Research Article

\title{
Convergence of Antiperiodic Boundary Value Problems for First-Order Integro-Differential Equations
}

\author{
Yameng Wang, ${ }^{1}$ Juan Zhang, ${ }^{1}$ and Yufeng Sun ${ }^{2}{ }^{2}$ \\ ${ }^{1}$ College of Mathematics and Information Science, Hebei University, Baoding 071002, China \\ ${ }^{2}$ School of Mathematics and Statistics, Shaoguan University, Shaoguan 512005, China
}

Correspondence should be addressed to Yufeng Sun; yufengsun@sgu.edu.cn

Received 19 March 2020; Revised 21 April 2020; Accepted 29 April 2020; Published 25 July 2020

Guest Editor: Thabet Abdeljawad

Copyright ( $\odot 2020$ Yameng Wang et al. This is an open access article distributed under the Creative Commons Attribution License, which permits unrestricted use, distribution, and reproduction in any medium, provided the original work is properly cited.

In this paper, we investigate the convergence of approximate solutions for a class of first-order integro-differential equations with antiperiodic boundary value conditions. By introducing the definitions of the coupled lower and upper solutions which are different from the former ones and establishing some new comparison principles, the results of the existence and uniqueness of solutions of the problem are given. Finally, we obtain the uniform and rapid convergence of the iterative sequences of approximate solutions via the coupled lower and upper solutions and quasilinearization method. In addition, an example is given to illustrate the feasibility of the method.

\section{Introduction}

In recent decades, the integro-differential equations have developed rapidly because the models described by various of integro-differential equations have appeared in a number of fields such as fluid dynamics, biology, economics, and control theory, for details and examples, we can refer to references [1-7] and cited therein. Meanwhile, the qualitative theory of integral differential equations creates an branch of nonlinear analysis. Boundary value problems for various first-order integral differential equations have been studied by several researchers, and there are some results on the existence of solutions and extremal solutions, the controllability problem controllability of integral boundary value conditions, and antiperiodic boundary value conditions, such as ordinary differential equations [8-12], difference equations $[13,14]$, fractional differential equations [15-20], impulsive differential equations $[9,14,21,22]$, integro-differential equations, and impulsive functional differential equations [18, 23-26].

However, we found that most of these known results concerned with the existence and uniformly convergence results of solutions and extremal solutions via the method of upper and lower solutions coupled with the monotone iterative technique (see [27]). It is well known that the method of quasilinearization (QSL) provides a powerful tool for obtaining convergence of approximate solutions of nonlinear problems [28, 29]. The technique of upper and lower solutions coupled with the QSL have been applied successfully to obtain monotone sequences of approximate solutions converging uniformly and quadratically to the unique solution of integro-differential equations with antiperiodic boundary value conditions [30-32]. In terms of applications, it is important to pay attention to the high-order convergence of sequences of approximate solutions. The high-order convergence results of various differential equations can be found in [33-39].

In this paper, we consider the following first-order integro-differential equations with antiperiodic boundary value conditions (APBVP):

$$
\left\{\begin{array}{l}
x^{\prime}(t)=f(t, x(t), \quad(T x)(t)), t \in J, \\
x(0)=-x(T),
\end{array}\right.
$$

where $f \in C\left(J \times R^{2}, R\right), J=[0, T],(T x)(t)=\int_{0}^{t} k(t, s) x$ $(s) \mathrm{d} s, k \in C\left(D, R_{+}\right), k_{0}=\sup \{k(t, s)\}, D=\{(t, s) \in J \times J: t$ $\geq s\}$. 
The aim of this paper is to investigate the convergence of approximate solutions of the problem. We give the particular definitions of the coupled lower and upper related solutions which are new and establish some new comparison principles in order to discuss the existence and uniqueness of the solutions. Then, by using the method of quasilinearization, we obtain the two monotone sequences of approximate solutions converging to the unique solution of the problem with rate of convergence of order $k$. Finally, we give an example to illustrate our main results.

\section{Comparison Theorems}

In this section, we begin with some comparison principles that will be useful in later discussions.

Lemma 1. Assume that there exist constants $M>0, N>0$, and $\gamma \geq 0$, such that

$$
\left(M+k_{0} \mathrm{NT}\right) T \leq 1 .
$$

If there exists a function $p \in C^{1}[J, R]$, such that

$$
\left\{\begin{array}{l}
p^{\prime}(t) \leq-M(p(t)+\gamma)-N(T p)(t), \quad t \in J, \\
p(0) \leq p(T),
\end{array}\right.
$$

then $p(t) \leq 0$ on $J$.

Proof. Suppose the conclusion is not true, and we consider the following two cases, where $p(0) \leq 0$ and $p(0)>0$, respectively.

Case 1. When $p(0) \leq 0$, there exists a $t^{*} \in(0, T]$, such that $p\left(t^{*}\right)>0$. Let $t_{*} \in\left[0, t^{*}\right]$, such that $p\left(t_{*}\right)=\inf p(t)=-b$, $b \geq 0$. By equation (3), we have

$$
p^{\prime}(t) \leq b\left[M+k_{0} \mathrm{TN}\right] \text {. }
$$

Integrating inequality (4) from $t_{*}$ to $t^{*}$, we obtain

$$
0<p\left(t^{*}\right) \leq p\left(t_{*}\right)+b \int_{t_{*}}^{t^{*}}\left\{M+k_{0} \mathrm{TN}\right\} \mathrm{d} t .
$$

Thus,

$$
b<b \int_{0}^{T}\left\{M+k_{0} \mathrm{TN}\right\} \mathrm{d} t
$$

which contradicts (2), therefore $p(t) \leq 0$.

Case 2. When $p(0)>0$, there are two cases: $p(t)>0$ for $t \in J$ or there exist $\bar{t}, \underline{t}$, such that $p(\underline{t}) \leq 0$ and $p(\bar{t})>0$ for $\bar{t}, \underline{t} \in J$.

Case 3. When $p(t)>0$, by equation (3), we have $p^{\prime}(t) \leq 0$, which contradicts the condition of equation (3).

Case 4. If there exist $\bar{t}$ and $\underline{t}$, such that $p(\underline{t}) \leq 0$ and $p(\bar{t})>0$, we have $q\left(\widetilde{t}_{*}\right)=\inf p(t)=-b$, where $\widetilde{t}_{*} \in(0, T), b \geq 0$. Then, equation (4) holds. Integrating inequality (4) from $\tilde{t}_{*}$ to $T$, we have

$$
0<p(\bar{t}) \leq p\left(\widetilde{t}_{*}\right)+b \int_{\tilde{t}_{*}}^{T}\left\{M+k_{0} \mathrm{TN}\right\} \mathrm{d} t,
$$

which is also a contradiction. The proof of Lemma 1 is completed.

Next, consider the linear APBVP:

$$
\left\{\begin{array}{l}
x^{\prime}(t)+M(x(t)+\gamma)+N(T x)(t)=0, \quad t \in J, \\
x(0)=-x(T) .
\end{array}\right.
$$

Corollary 1. Assume that $M>N>0$ and $\left(M+k_{0} N T\right) T \leq 1$, then APBVP (8) has at most one solution.

Proof. Let $x_{1}, x_{2}$ be any solution of $\operatorname{APBVP}(8), x_{1} \geq x_{2}$, and $y(t)=x_{1}(t)-x_{2}(t)$, then

$$
\left\{\begin{array}{l}
y^{\prime}(t)+M y(t)+N(T y)(t)=0 \\
y(0)=-y(T)
\end{array}\right.
$$

If $y(T)>0$, then it follows from (9) that $y(0)<0$. By Lemma 1 , we have $y(t) \leq 0$, that is a contradiction. On the contrary, if $y(T)<0$, we have $y(0)>0$. By the proof of Lemma 1 , we have $y(t) \leq 0$, that is also a contradiction. Therefore, we have $y(T)=y(0)=0$. Furthermore, by Lemma 1 , we have $y(t) \leq 0$, that is, $y(t)=0$ for $t \in J$. The proof of Corollary 1 is completed.

Similar to the proof of Lemma 1, we have the following lemma.

Lemma 2. Assume that there exist integrable functions $\phi_{i}(t)<0, i=1,2$, such that

$$
\int_{0}^{T}\left\{\phi_{1}(t)+\phi_{2}(t) k_{0} T\right\} \mathrm{d} t \geq-1 .
$$

If there exist functions $p_{i} \in C^{1}[J, R], i=1,2$, such that $p_{1}^{\prime}(t) \leq \phi_{1}(t)\left(p_{1}(t)+\gamma_{1}\right)+\phi_{2}(t)\left(T p_{1}\right)(t), \quad$ for $t \in J, p_{1}(0) \leq p_{2}(T)$, $p_{2}^{\prime}(t) \leq \phi_{1}(t)\left(p_{2}(t)+\gamma_{2}\right)+\phi_{2}(t)\left(T p_{2}\right)(t), \quad$ for $t \in J, p_{2}(0) \leq p_{1}(T)$,

where

$$
\begin{aligned}
& \gamma_{1}=\frac{p_{1}(T)-p_{2}(0)}{1-e \int_{0}^{T} \phi_{1}(t) \mathrm{d} t}, \\
& \gamma_{2}=\frac{p_{2}(T)-p_{1}(0)}{1-e \int_{0}^{T} \phi_{2}(t) \mathrm{d} t} .
\end{aligned}
$$

Then, $p_{1}(t) \leq 0$ and $p_{2}(t) \leq 0$ on $J$. 
Proof. We just prove that the case of $p_{1}(t) \leq 0$. Suppose that the conclusion is not true, we can consider the following two cases, where $p_{1}(0) \leq 0$ and $p_{1}(0)>0$, respectively.

Case 5. When $p_{1}(0) \leq 0$, by the proof of Lemma 1 , we have $\int_{0}^{T}\left\{\phi_{1}(t)+\phi_{2}(t) k_{0} T\right\} \mathrm{d} t<-1$, which contradicts (10).

Case 6. When $p_{1}(0)>0$, there are two cases: $p_{1}(t)>0$ for $t \in J$ or there exist $\bar{t}$ and $\underline{t}$, such that $p_{1}(\underline{t}) \leq 0$ and $p_{1}(\bar{t})>0$ for $\bar{t}, \underline{t} \in J$.

Case 7. When $p_{1}(t)>0, t \in J$, if $p_{2}(0) \leq 0$, by the proof of Lemma 1 , we have $p_{2}(t) \leq 0$, that implies $p_{1}(0) \leq p_{2}(T) \leq 0$, which is a contradiction.

If $p_{2}(0)>0$, we have $p_{2}(T) \geq p_{1}(0)>0$. Then, there are two cases: $p_{2}(t)>0$ for $t \in J$ and there exist $\tilde{t}$ and $\widehat{t}$, such that $p_{2}(\widetilde{t}) \leq 0$ and $p_{2}(\widehat{t})>0$, respectively.

Case 8. When $p_{2}(t)>0$ for all $t \in J$, we have $p_{2}^{\prime}(t)<0$; hence, $p_{2}(t)$ is decreasing. By $p(t)>0$ and equation (11), imply $p_{1}^{\prime}(t)<0$; then, $p_{1}(t)$ is decreasing and $p_{2}(0)>p_{2}(T) \geq p_{1}(0)>p_{1}(T) \geq p_{2}(0)$, which is a contradiction.

Case 9. For another case, we have $p_{2}\left(\widetilde{t}_{*}\right)=\inf p_{2}(t)=-b$, where $\widetilde{t}_{*} \in(0, T), b \geq 0$. Equation (11) implies that

$$
p_{2}^{\prime}(t) \leq(-b)\left[\phi_{1}(t)+k_{0} T \phi_{2}(t)\right] .
$$

Integrating inequality (13) from $\widetilde{t}_{*}$ to $T$, we have

$$
0<p_{2}(T) \leq p_{2}\left(\widetilde{t}_{*}\right)-b \int_{\widetilde{t}_{*}}^{T}\left\{\phi_{1}(t)+k_{0} T \phi_{2}(t)\right\} \mathrm{d} t .
$$

Thus,

$$
b<-b \int_{0}^{T}\left\{\phi_{1}(t)+k_{0} T \phi_{2}(t)\right\} \mathrm{d} t,
$$

which is also a contradiction.

The proof of Case 4 is analogous to the proof of Lemma 1 , and we omit its details here. This completes the proof of Lemma 2.

Remark 1. When $\gamma=0$ and $\gamma_{i}=0, i=1,2$, respectively, the conclusion of Lemmas 1 and 2 is also true.

\section{Linear APBVP}

In this section, we consider the linear APBVP:

$$
\left\{\begin{array}{l}
x^{\prime}(t)+M x(t)+N(T x)(t)=\sigma(t), \quad t \in J, \\
x(0)+x(T)=0 .
\end{array}\right.
$$

We can get the result of the existence and unique solution of equation (16).

Theorem 1. Assume that $M, N>0, \quad M>2 N T$, and $\left(M+k_{0} N T\right) T \leq 1$. Then, APBVP (16) possesses a unique solution.

Proof. For any $x \in C(J, R)$, denoting $\|x\|=\max _{t \in J}|x(t)|$. Let

$$
\begin{aligned}
& \omega_{0}=\max _{t \in J}|\sigma(t)|, \\
& \omega_{1}=\frac{2\left(1-e^{-\mathrm{MT}}\right) \omega_{0}}{M-2 \mathrm{NT}} .
\end{aligned}
$$

We define an operator $S: E \longrightarrow C(J, R)$ as follows:

$$
\begin{aligned}
(S x)(t)= & \frac{e^{-M(t+T)}}{e^{-M T}+1} \int_{0}^{T}(\sigma(s)-N(T x)(s)) e^{M s} \mathrm{~d} s \\
& +e^{-M t} \int_{0}^{t}(\sigma(s)-N(T x)(s)) e^{M s} \mathrm{~d} s,
\end{aligned}
$$

where $E=\left\{x \in C(J, R):|x| \leq \omega_{1}, x(0)-x(T)=0\right\}$.

It is easy to see that $E$ is a closed, bounded, and convex set. Furthermore, for any $x \in E$, we have

$$
\begin{aligned}
|(S x)(t)| \leq & \frac{e^{-M(t+T)}}{e^{-M T}+1} \int_{0}^{T}(|\sigma(s)|+N|(T x)(s)|) e^{M s} \mathrm{~d} s, \\
& +e^{-M t} \int_{0}^{T}(|\sigma(s)|+N|(T x)(s)|) e^{M s} \mathrm{~d} s \\
\leq & \frac{e^{-M(t+T)}}{e^{-M T}+1} \int_{0}^{T}\left(\omega_{0}+\mathrm{NT} \omega_{1}\right) e^{M s} \mathrm{~d} s \\
& +e^{-M t} \int_{0}^{t}\left(\omega_{0}+N T \omega_{1}\right) e^{M s} \mathrm{~d} s \\
\leq & \frac{2\left(\omega_{0}+N \omega_{1} T\right)\left(1-e^{-M T}\right)}{M}=\omega_{1}
\end{aligned}
$$

which implies that $\|S(x)\| \leq \omega_{1}$, that is, $S(E) \subset E$ and $S$ is uniformly bounded. Furthermore, for any $t_{1}, t_{2} \in J$, we have 


$$
\begin{aligned}
\left|(S x)\left(t_{1}\right)-(S x)\left(t_{2}\right)\right|= & \mid \frac{e^{-M\left(t_{1}+T\right)}}{e^{-M T}+1} \int_{0}^{T}(|\sigma(s)|-N|(T x)(s)|) e^{M s} \mathrm{~d} s \\
& -\frac{e^{-M\left(t_{2}+T\right)}}{e^{-M T}+1} \int_{0}^{T}(|\sigma(s)|-N|(T x)(s)|) e^{M s} \mathrm{~d} s \\
& +e^{-M t_{1}} \int_{0}^{t_{1}}(|\sigma(s)|-N|(T x)(s)|) e^{M s} \mathrm{~d} s \\
& -e^{-M t_{2}} \int_{0}^{t_{2}}(|\sigma(s)|-N|(T x)(s)|) e^{M s} \mathrm{~d} s \mid \\
\leq & \frac{\mid e^{-M\left(t_{1}+T\right)}-e^{-M\left(t_{2}+T\right) \mid} \int_{0}^{T}(|\sigma(s)|+N|(T x)(s)|) e^{M s} \mathrm{~d} s}{e^{-M T}+1} \\
& +\left|e^{-M t_{1}}-e^{-M t_{2}}\right| \int_{0}^{t_{1}}(|\sigma(s)|+N|(T x)(s)|) e^{M s} \mathrm{~d} s \\
& +e^{-M t_{2}} \int_{\min \left\{t_{1}, t_{2}\right\}}^{\max \left\{t_{1}, t_{2}\right\}}(|\sigma(s)|+N|(T x)(s)|) e^{M s} \mathrm{~d} s .
\end{aligned}
$$

Since $\sigma$ and $x$ are bounded, thus $S$ is uniformly continuous. According to Ascoli-Arzela's theorem, there exists the subsequences $\left\{S x_{n}\right\}$ converging uniformly on $J$ to the continuous functions $S x$ and $S x \in E$, then, we can see that $S$ is compact. Therefore, there exists a solution of APBVP (16) by Schauder's fixed point theorem. The uniqueness of solutions of APBVP (16) follows from Corollary 1. The proof is completed.

\section{Nonlinear APBVP}

In this section, we give the existence and uniqueness of the solutions of APBVP (1).

Definition 1. The functions $v, w \in C^{\prime}(J, R)$ are said to be a pair of coupled lower and upper solutions for APBVP (1) if the following inequalities

$$
\begin{aligned}
& \left\{\begin{array}{l}
v^{\prime}(t) \leq f(t, v(t), \quad(T v)(t))-M \gamma_{1}, \\
v(0) \leq-w(T),
\end{array}\right. \\
& \left\{\begin{array}{l}
w^{\prime}(t) \geq f(t, w(t),(T w)(t))+M \gamma_{2} \\
w(0) \geq-v(T),
\end{array}\right.
\end{aligned}
$$

hold, where $M>0, \gamma_{1}=(v(T)+w(0)) /\left(1-e^{-M T}\right)$, and $\gamma_{2}=(-w(T)-v(0)) /\left(1-e^{-M T}\right)$.

Theorem 2. Assume that the following conditions hold.

$\left(H_{1}\right) v, w \in C^{\prime}(J, R)$ are a pair of coupled lower and upper solutions of APBVP (1) such that $v \leq w$ on $J$;

$\left(H_{2}\right)$ There exist constants $M>0$ and $N>0$ such that $M>2 \mathrm{NT} \geq 0,\left(M+k_{0} \mathrm{NT}\right) T \leq 1$, and

$$
|f(t, \bar{u}, T \bar{u})-f(t, u, T u)| \leq M(\bar{u}-u)+N(T \bar{u}-T u),
$$

while $v \leq u \leq \bar{u} \leq w$ and $T v \leq T u \leq T \bar{u} \leq T w$ for $t \in J$.

Then, APBVP (1) has a unique solution $x \in[v, w]$.

Proof. We construct iterative sequences $\left\{v_{n}\right\},\left\{w_{n}\right\}$ $\subset C^{\prime}(J, R)$ as follows, $v_{1}=v$ and $w_{1}=w$ on $J$, and for $n>1$, $v_{n}$ and $w_{n}$ are the solutions of

$$
\begin{aligned}
& \left\{\begin{array}{l}
v_{n}^{\prime}(t)=f\left(t, v_{n-1}(t),\left(T v_{n-1}\right)(t)\right)-M\left[v_{n}(t)-v_{n-1}(t)\right]-N\left[\left(T v_{n}\right)(t)-\left(T v_{n-1}\right)(t)\right], \\
v_{n}(0)=-w_{n}(T),
\end{array}\right. \\
& \left\{\begin{array}{l}
w_{n}^{\prime}(t)=f\left(t, w_{n-1}(t),\left(T w_{n-1}\right)(t)\right)-M\left[w_{n}(t)-w_{n-1}(t)\right]-N\left[\left(T w_{n}\right)(t)-\left(T w_{n-1}\right)(t)\right], \\
w_{n}(0)=-v_{n}(T) .
\end{array}\right.
\end{aligned}
$$


The existence and uniqueness of the solution can be obtained by standard arguments for IVP (24) and (25).

We next prove that $v_{1} \leq v_{2} \leq w_{2} \leq w_{1}$.
Let $p_{1}=v_{1}-v_{2}$ and $p_{2}=w_{2}-w_{1}$, by the condition of $\left(H_{2}\right)$, and we have $p_{1}(0) \leq p_{2}(T), p_{2}(0) \leq p_{1}(T)$, and

$$
\left\{\begin{array}{l}
p_{1}^{\prime}(t) \leq-M\left[v_{1}(t)-v_{2}(t)+\gamma_{1}\right]-N\left[\left(T v_{1}\right)(t)-\left(T v_{2}\right)(t)\right]=-M\left(p_{1}(t)+\gamma_{1}\right)-N\left(T p_{1}\right)(t), \\
p_{2}^{\prime}(t) \leq-M\left[w_{2}(t)-w_{1}(t)+\gamma_{2}\right]-N\left[\left(T w_{2}\right)(t)-\left(T w_{1}\right)(t)\right]=-M\left(p_{2}(t)+\gamma_{2}\right)-N\left(T p_{2}\right)(t)
\end{array}\right.
$$

where $\quad \gamma_{1}=\left(p_{1}(T)-p_{2}(0)\right) /\left(1-e^{-M T}\right) \quad$ and $\gamma_{2}=\left(p_{2}(T)-p_{1}(0)\right) /\left(1-e^{-M T}\right)$.

By Lemma 2 , we have $p_{1}=v_{1}-v_{2} \leq 0$ and $p_{2}=w_{2}-$ $w_{1} \leq 0$ on $J$.

Let $p=v_{2}-w_{2}$, by the condition of $\left(\mathrm{H}_{2}\right)$, and we have

$$
p^{\prime}(t) \leq-M p(t)-\mathrm{NT} p(t), \quad p(0)=p(T) .
$$

By using similar arguments of Lemma 1, we have $p=v_{2}-w_{2} \leq 0$. Therefore, it is easy to see that these sequences satisfy

$$
v_{n} \leq v_{n+1} \leq w_{n+1} \leq w_{n}, n \geq 1 .
$$

Then, we have two monotone sequences which are bounded, and there exist $\rho$ and $\mu$, which satisfy $\lim _{n \longrightarrow \infty} v_{n}=\rho, \lim _{n \longrightarrow \infty} w_{n}=\mu$, and $\rho \leq \mu$. Moreover, the convergence is uniform on $J$.

Set $p=\mu-\rho$, then we obtain

$$
\left\{\begin{array}{l}
p^{\prime}(t) \leq-M p(t)-N(T p)(t) \\
p(0)=p(T)
\end{array}\right.
$$

By Lemma 1, we have $p(t) \leq 0$ for $t \in J$. Hence, $\rho \equiv \mu$ for $t \in J$, and we can conclude $\rho \equiv \mu \equiv x$, in which $x$ is the solution of APBVP (1). The proof of Theorem 2 is completed.

\section{Quasilinearization}

In this section, we apply the quasilinesrization method in order to obtain the result on convergence of the iterative sequences of approximate solutions for APBVP (1).

Consider the Banach space $C(J, R)$ with the usual maximum norm $\|x\|_{1}=\max _{t \in J}|x(t)|$. For any $x \in C(J, R)$, we call that a given sequence $\left\{x_{n}\right\}$ converges to $x$ with order of convergence $k$, if $\left\{x_{n}\right\}$ converges to $x$ in $C(J, R)$ and there exist $n_{0} \in \mathrm{N}$ and $k>0$ such that $\left\|x_{m+1}-x\right\|_{1} \leq\left\|x_{m}-x\right\|_{1}^{k}$ for all $m \geq n_{0}$.

Theorem 3. Assume that the conditions of $\left(H_{1}\right)-\left(H_{2}\right)$ hold.

$\left(H_{3}\right)\left(\partial^{i} f / \partial x^{i}\right)$ and $\partial^{i} f / \partial(T x)^{i}$ exist and are continuous for $i=0,1, \ldots, k$, and

$$
\begin{aligned}
& \sum_{i=1}^{k} i M_{i}|w-v|^{i-1} \leq M, \\
& \sum_{i=1}^{k} i N_{i}|w-v|^{i-1} \leq N,
\end{aligned}
$$

where $M_{i}$ and $N_{i}$ are constants with

$$
\begin{aligned}
\left\|\frac{\partial^{i} f}{\partial x^{i}}(t, u, T u)\right\| & \leq(i !) M_{i}, \\
\left\|\frac{\partial^{i} f}{\partial(T x)^{i}}(t, u, T u)\right\| & \leq(i !) N_{i},
\end{aligned}
$$

where $(t, u, T u) \in \Omega=\{(t, u, T u): v \leq u \leq w\}$.

Then, there exist monotone sequences $\left\{v_{n}\right\},\left\{w_{n}\right\}$ of approximate solutions converging to the unique solution of (1) with rate of convergence of order $k$.

Proof. Let the function

$$
\begin{aligned}
f(t, u, T u)= & \sum_{i=0}^{k-1} \frac{\partial^{i} f}{\partial x^{i}}(t, \alpha, T \alpha) \frac{(u-\alpha)^{i}}{i !}+\frac{\partial^{k} f}{\partial x^{k}}(t, \chi, T \chi) \frac{(u-\alpha)^{k}}{k !} \\
& +\sum_{i=0}^{k-1} \frac{\partial^{i} f}{\partial(T x)^{i}}(t, \alpha, T \alpha) \frac{T(u-\alpha)^{i}}{i !} \\
& +\frac{\partial^{k} f}{\partial(T x)^{k}}(t, \chi, T \chi) \frac{T(u-\alpha)^{k}}{k !} \\
\equiv & g(t, u, T u ; \alpha, T \alpha),
\end{aligned}
$$

where $v \leq \alpha \leq u \leq w, \chi \in[\alpha, u]$. Consider the following linear equation:

$$
\left\{\begin{array}{l}
u^{\prime}(t)=g(t, u, T u ; \alpha, T \alpha), \quad t \in J, \\
u(0)=-u(T)
\end{array}\right.
$$

Setting $v_{0}=v$, by $\left(H_{3}\right)$, we have

$$
\left\{\begin{array}{l}
v_{0}^{\prime}(t) \leq f\left(t, v_{0}(t),\left(T v_{0}\right)(t)\right)-M \gamma_{1} \equiv g\left(t, v_{0}(t),\left(T v_{0}\right)(t) ; v_{0}(t),\left(T v_{0}\right)(t)\right)-M \gamma_{1}, \\
v_{0}(0) \leq-w_{0}(T)
\end{array}\right.
$$


Similarly, setting $w_{0}=w$, we obtain

$$
\left\{\begin{array}{l}
w_{0}^{\prime}(t) \geq f\left(t, w_{0}(t),\left(T w_{0}\right)(t)\right)+M \gamma_{2} \equiv g\left(t, w_{0}(t),\left(T w_{0}\right)(t) ; w_{0}(t),\left(T w_{0}\right)(t)\right)+M \gamma_{2} \\
w_{0}(0) \geq-v_{0}(T)
\end{array}\right.
$$

Then, $v_{0}$ and $w_{0}$ are lower and upper solutions of equation (33), respectively. Furthermore, for $t \in J$ and $v \leq x \leq y \leq w$, we have

$$
\begin{aligned}
\mid g( & t, x, T x ; \alpha, T \alpha)-g(t, y, T y ; \alpha, T \alpha) \mid \\
= & \mid \sum_{i=0}^{k-1} \frac{\partial^{i} f}{\partial x^{i}}(t, \alpha, T \alpha)\left(\frac{(x-\alpha)^{i}-(y-\alpha)^{i}}{i !}\right) \\
& +\frac{\partial^{k} f}{\partial x^{k}}(t, \chi, T \chi)\left(\frac{(x-\alpha)^{k}-(y-\alpha)^{k}}{k !}\right) \\
& +\sum_{i=0}^{k-1} \frac{\partial^{i} f}{\partial(T x)^{i}}(t, \alpha, T \alpha)\left(\frac{T(x-\alpha)^{i}-T(y-\alpha)^{i}}{i !}\right) \\
& +\frac{\partial^{k} f}{\partial(T x)^{k}}\left(t, \chi, T \chi\left(\frac{T(x-\alpha)^{k}-T(y-\alpha)^{k}}{k !}\right) \mid\right. \\
= & |x-y| \mid \sum_{i=0}^{k-1} \frac{\partial^{i} f}{\partial x^{i}}(t, \alpha, T \alpha)\left(\frac{1}{i !} \sum_{j=0}^{i-1}(x-\alpha)^{i-1-j}(y-\alpha)^{j}\right) \\
\leq & M|x-y|+N T|x-y| . \\
& +\frac{\partial^{k} f}{\partial(T x)^{k}}\left(t, \chi, T \chi\left(\frac{T(x-\alpha)^{i-1-j} T(y-\alpha)^{j}}{\partial x^{k}}\right) \mid\right. \\
& +T|x-y| \mid \sum_{i=0}^{k} \frac{\partial^{i} f}{\partial(T x)^{i}}(t, \alpha, T \alpha)\left(\frac{1}{i !} \sum_{j=0}^{i-1} T(x-\alpha)^{i-1-j} T(y-\alpha)^{j}\right) \\
k ! & (x-\alpha)^{i-1-j}(y-\alpha)^{j} \\
& +\sum_{i=1}^{k} M_{i}\left(\sum_{j=0}^{i-1}(y-x)^{i-1}\right)+T|x-y| \sum_{i=1}^{k} N_{i}\left(\sum_{j=0}^{i-1} T(y-x)^{i-1}\right)
\end{aligned}
$$

Using Theorem 2, we know that problem (33) has a solution in $[v, w]$.

Let $v_{1}$ be a solution of the mentioned problem, with $v_{1} \in[v, w]$, and we suppose $v=v_{0} \leq v_{1} \leq \cdots \leq v_{n} \leq w$, where $v_{n} \in\left[v_{n-1}, w\right]$ is the solution of

$$
\left\{\begin{array}{l}
u^{\prime}(t)=g\left(t, u, T u ; v_{n-1}, T v_{n-1}\right), \quad t \in J \\
u(0)=-u(T)
\end{array}\right.
$$

where $v_{n-1}$ and $w$ are lower and upper solutions, respectively, for the following problem: 


$$
\left\{\begin{array}{l}
u^{\prime}(t)=g\left(t, u, T u ; v_{n}, T v_{n}\right), \quad t \in J \\
u(0)=-u(T)
\end{array}\right.
$$

Similarly, we know that $g\left(t, u, T u ; v_{n}, T v_{n}\right)$ satisfies the conditions of Theorem 2, then problem (33) has a solution in $\left[v_{n}, w\right]$. Let $v_{n+1}$ be a solution of the mentioned problem, with $v_{n+1} \in\left[v_{n}, w\right]$. Hence, the constructed sequence $\left\{v_{n}\right\}$ is nondecreasing and bounded. In the same way, we can construct the sequence $\left\{w_{n}\right\}$ which is nonincreasing and bounded. Therefore, we obtain the two monotone sequences converge uniformly.

Let $\lim v_{n}=\rho$ and $\lim w_{n}=\mu$, and we can get $\rho \equiv \mu \equiv u$ by using Theorem 2, in which $u$ is the solution of (1).

Now, we show that the convergence of $\left\{v_{n}\right\},\left\{w_{n}\right\}$ to $u$ is of order $k$. Let

$$
\begin{aligned}
& p_{n}=u-v_{n} \geq 0, \\
& q_{n}=w_{n}-u \geq 0 .
\end{aligned}
$$

Firstly, we note that

$$
\begin{aligned}
u \prime(t)= & \sum_{i=0}^{k-1} \frac{\partial^{i} f}{\partial x^{i}}\left(t, v_{n}, T v_{n}\right) \frac{\left(u-v_{n}\right)^{i}}{i !}+\frac{\partial^{k} f}{\partial x^{k}}\left(t, \chi_{n}, T \chi_{n}\right) \frac{\left(u-v_{n}\right)^{k}}{k !} \\
& +\sum_{i=0}^{k-1} \frac{\partial^{i} f}{\partial(T x)^{i}}\left(t, v_{n}, T v_{n}\right) \frac{T\left(u-v_{n}\right)^{i}}{i !} \\
& +\frac{\partial^{k} f}{\partial(T x)^{k}}\left(t, \chi_{n}, T \chi_{n}\right) \frac{T\left(u-v_{n}\right)^{k}}{k !}
\end{aligned}
$$

where $\chi_{n} \in\left[v_{n}, u\right]$. In sequence,

$$
\begin{aligned}
& p_{n+1}^{\prime}(t)=\sum_{i=0}^{k-1} \frac{\partial^{i} f}{\partial x^{i}}\left(t, v_{n}, T v_{n}\right)\left(\frac{p_{n}^{i}-\left(v_{n+1}-v_{n}\right)^{i}}{i !}\right)+\frac{\partial^{k} f}{\partial x^{k}}\left(t, \chi_{n}, T \chi_{n}\right)\left(\frac{p_{n}^{k}-\left(v_{n+1}-v_{n}\right)^{k}}{k !}\right) \\
& +\sum_{i=0}^{k-1} \frac{\partial^{i} f}{\partial(T x)^{i}}\left(t, v_{n}, T v_{n}\right)\left(\frac{T p_{n}^{i}-T\left(v_{n+1}-v_{n}\right)^{i}}{i !}\right)+\frac{\partial^{k} f}{\partial(T x)^{k}}\left(t, \chi_{n}, T \chi_{n}\right)\left(\frac{T p_{n}^{k}-T\left(v_{n+1}-v_{n}\right)^{k}}{k !}\right) \\
& =p_{n+1} \sum_{i=1}^{k-1} \frac{\partial^{i} f}{\partial x^{i}}\left(t, v_{n}, T v_{n}\right)\left(\frac{1}{i !} \sum_{j=0}^{i-1} p_{n}^{i-1-j}\left(v_{n+1}-v_{n}\right)^{j}\right)+\frac{\partial^{k} f}{\partial x^{k}}\left(t, \chi_{n}, T \chi_{n}\right)\left(\frac{p_{n}^{k}-\left(v_{n+1}-v_{n}\right)^{k}}{k !}\right) \\
& +T p_{n+1} \sum_{i=1}^{k-1} \frac{\partial^{i} f}{\partial x^{i}}\left(t, v_{n}, T v_{n}\right)\left(\frac{1}{i !} \sum_{j=0}^{i-1}\left(T p_{n}^{i-1-j} T\left(v_{n+1}-v_{n}\right)^{j}\right)+\frac{\partial^{k} f}{\partial(T x)^{k}}\left(t, \chi_{n}, T \chi_{n}\right)\left(\frac{T p_{n}^{k}-T\left(v_{n+1}-v_{n}\right)^{k}}{k !}\right) .\right.
\end{aligned}
$$

In view of condition $\left(H_{3}\right)$, by the continuity of $\partial^{i} f / \partial x^{i}$ and $\partial^{i} f / \partial(T x)^{i}$ in $\Omega$, we have

$$
p_{n+1}^{\prime}(t) \leq A p_{n+1}(t)+\mathrm{BT} p_{n+1}(t)+C p_{n}^{k}(t)+\mathrm{DT} p_{n}^{k}(t),
$$

where $C=2 M_{k}, D=2 N_{k}$, and

$$
\begin{aligned}
& \sum_{i=0}^{k-1} \frac{\partial^{i} f}{\partial x^{i}}\left(t, v_{n}, T v_{n}\right)\left(\frac{1}{i !} \sum_{j=0}^{i-1} p_{n}^{i-1-j}\left(v_{n+1}-v_{n}\right)^{j}\right) \leq A, \\
& \sum_{i=0}^{k-1} \frac{\partial^{i} f}{\partial x^{i}}\left(t, v_{n}, T v_{n}\right)\left(\frac{1}{i !} \sum_{j=0}^{i-1} T p_{n}^{i-1-j} T\left(v_{n+1}-v_{n}\right)^{j}\right) \leq B .
\end{aligned}
$$

Similarly, we have

$$
q_{n+1}^{\prime}(t) \leq A q_{n+1}(t)+\mathrm{BT} q_{n+1}(t)+C q_{n}^{k}(t)+\mathrm{DT} q_{n}^{k}(t) \text {. }
$$

The boundary conditions are

$$
\begin{aligned}
& p_{n}(0)=q_{n}(T), \\
& q_{n}(0)=p_{n}(T) .
\end{aligned}
$$

Let $R_{n}=p_{n}+q_{n}$, and we obtain

$$
\left\{\begin{array}{l}
R_{n+1}^{\prime}(t) \leq \mathrm{AR}_{n+1}(t)+\mathrm{BTR}_{n+1}(t)+C\left(p_{n}^{k}(t)+q_{n}^{k}(t)\right)+\mathrm{DT}\left(p_{n}^{k}(t)+q_{n}^{k}(t)\right) \leq\left(A+k_{0} B\right) R_{n+1}(t)+\left(C+k_{0} D\right)\left(p_{n}^{k}(t)+q_{n}^{k}(t)\right) \\
R_{n+1}(0)=R_{n+1}(T) .
\end{array}\right.
$$


Using Gronwall's inequality for (46), we have

$$
\begin{gathered}
R_{n+1}(t) \leq e^{\left(A+k_{0} B\right) t} R_{n+1}(0)+\int_{0}^{t} e^{\left(A+k_{0} B\right)(t-s)} \\
\left(C+k_{0} D\right)\left(p_{n}^{k}(s)+q_{n}^{k}(s)\right) \mathrm{d} s .
\end{gathered}
$$

Let $t=T$, and we have

$$
\begin{gathered}
R_{n+1}(0) \leq e^{\left(A+k_{0} B\right) T} R_{n+1}(0)+\frac{T}{A+k_{0} B} e^{\left(A+k_{0} B\right) T} \\
\cdot\left(C+k_{0} D\right) \max _{t \in J}\left(p_{n}^{k}(t)+q_{n}^{k}(t)\right),
\end{gathered}
$$

which implies

$$
R_{n+1}(0) \leq \frac{e^{\left(A+k_{0} B\right) T}\left(C+k_{0} D\right) \max _{t \in J}\left(p_{n}^{k}(t)+q_{n}^{k}(t)\right)}{T\left(1-e^{\left(A+k_{0} B\right) T}\right)},
$$

that is,

$$
\max _{t \in J} R_{n+1}(t) \leq K\left(\max _{t \in J} p_{n}^{k}(t)+\max _{t \in J} q_{n}^{k}(t)\right),
$$

where $K=e^{\left(A+k_{0} B\right) T}\left(e^{\left(A+k_{0} B\right)} T\left(C+k_{0} D\right) \max _{t \in J} R_{n}^{k}(t)\right) / T$ $\left(1-e^{\left(A+k_{0} B\right) T}\right)+T /\left(A+k_{0} B\right) e^{\left(A+k_{0} B\right) T}\left(C+k_{0} D\right)$.

Since

$\max _{t \in J} p_{n}(t) \leq \max _{t \in J} R_{n}(t)$ and $\max _{t \in J} q_{n}(t) \leq \max _{t \in J} R_{n}(t)$, we get the desired convergence. The proof is completed.

\section{An Example}

In this section, we will provide an example which demonstrates the application of Theorem 3.

Example 1. Consider the following APBVP:

$$
\left\{\begin{array}{l}
x^{\prime}(t)=\frac{1}{20} t\left(1+x^{2}(t)\right)-\frac{1}{10} x-\frac{1}{20} \int_{0}^{t} x(s) \cos s \mathrm{~d} s, \quad t \in[0,1], \\
x(0)=-x(1)
\end{array}\right.
$$

It is easy to check that $v_{0}=-1$ and $w_{0}=1$ are lower and upper solutions of (51), respectively, which satisfies condition $\left(H_{1}\right)$ of Theorem 3. And we can show that

$$
\begin{aligned}
\left|f_{x}\right| & \leq \frac{1}{5}, \\
\left|f_{y}\right| & \leq \frac{1}{20}, \\
\left|f_{x x}\right| & \leq \frac{1}{10}, \\
f_{y y} & =0, \frac{\partial^{i} f}{\partial x^{i}}=0, \\
\frac{\partial^{i} f}{\partial(T x)^{i}} & =0, \\
i & =3,4, \ldots, k .
\end{aligned}
$$

Setting $\quad M_{1}=1 / 5, \quad M_{2}=1 / 10, \quad N_{1}=1 / 20, \quad N_{2}=0$, $M_{i}=0, \quad N_{i}=0, \quad i=3,4, \ldots, k, M=3 / 5$, and $N=1 / 20$, satisfy conditions $\left(\mathrm{H}_{2}\right)$ and $\left(\mathrm{H}_{3}\right)$ of Theorem 3. Then, convergence of the iterative sequences of approximate solutions for APBVP (51) are of order $k \geq 2$.

\section{Conclusion}

In this paper, we discussed the problem of rapid convergence for the first-order integro-differential equations with antiperiodic boundary value conditions. By using the particular definitions of the coupled lower and upper related solutions, which are new and some new comparison principles, we obtained the existence and uniqueness of solution of the problems. Meanwhile, by using the method of quasilinearization, we obtained the monotone sequences of approximate solutions, converging to the unique solution of such problems with the rate of convergence of order $k$. Finally, we give an example to illustrate our main results.

\section{Data Availability}

No data were used to support the findings of this study.

\section{Conflicts of Interest}

The authors declare that they have no competing interest.

\section{Authors' Contributions}

All authors read and approved the final manuscript.

\section{Acknowledgments}

This paper was supported by the National Natural Science Foundation of China (11771115) and Scientific Research start-up project of Shaoguan University (99000608).

\section{References}

[1] M. A. Alqudah, C. Ravichandran, T. Abdeljawad, and N. Valliammal, "New results on Caputo fractional-order neutral differential inclusions without compactness," Advances in Difference Equations, vol. 2019, no. 12, pp. 1-14, 2019.

[2] K. Jothimani, K. Kaliraj, Z. Hammouch, and C. Ravichandran, "New results on controllability in the framework of fractional integrodifferential equations with nondense domain," The European Physical Journal Plus, vol. 134, no. 9, pp. 1-10, 2019.

[3] M. Kostic, Abstract Volterra Integro-Differential Equations, Taylor \& Francis Group, LLC, Abingdon, UK, 2015.

[4] V. Lakshmikantham and M. Rama Mahana Rao, Theory of Lntegro-Differential Equations, Gordon and Breach Science Publishers, London, UK, 1995.

[5] J. D. Murray, Mathematical Biology, Springer, NewYork, NY, USA, second edition, 1993.

[6] C. Ravichandran, N. Valliammal, and J. J. Nieto, "New results on exact controllability of a class of fractional neutral integrodifferential systems with state-dependent delay in Banach spaces," Journal of the Franklin Institute, vol. 356, no. 3, pp. 1535-1565, 2019. 
[7] N. Valliammal, C. Ravichandran, and J. H. Park, "On the controllability of fractional neutral integrodifferential delay equations with nonlocal conditions," Mathematical Methods in the Applied Sciences, vol. 40, no. 14, pp. 5044-5055, 2017.

[8] Y. Chen, J. J. Nieto, and D. O’Regan, “Anti-periodic solutions for fully nonlinear first-order differential equations," Mathematical and Computer Modelling, vol. 46, pp. 1183-1190, 2007.

[9] W. Ding, Y. Xing, and M. Han, "Anti-periodic boundary value problems for first order impulsive functional differential equations," Applied Mathematics and Computation, vol. 186, no. 1, pp. 45-53, 2007.

[10] K. Z. Wang, "A new existence result for nonlinear first-order anti-periodic boundary value problems," Applied Mathematics Letters, vol. 21, pp. 1149-1154, 2008.

[11] S. Aizicovici, S. Reich, and S. Reich, "Anti-periodic solutions to a class of non-monotone evolution equations," Discrete \& Continuous Dynamical Systems-A, vol. 5, no. 1, pp. 35-42, 1999.

[12] D. Franco, J. J. Nieto, and D. ORegan, "Anti-periodic boundary value problem for nonlinear first order ordinary differential equations," Mathematical Inequalities \& Applications, vol. 6, pp. 477-485, 2003.

[13] R. P. Agarwal, A. Cabada, V. Otero-Espinar, and S. Dontha, "Existence and uniqueness of solutions for anti-periodic difference equations," Advances in Difference Equations, vol. 2004, no. 2, pp. 397-411, 2004.

[14] P. G. Wang and W. L. Wang, "Anti-periodic boundary value problem for first order impulsive delay difference equations," Advances in Difference Equations, vol. 2015, no. 1, 13 pages, 2015.

[15] P. Bedi, A. Kumar, T. Abdeljawad, and A. Khan, "Existence of mild solutions for impulsive neutral Hilfer fractional evolution equations," Advances in Difference Equations, vol. 2020, no. 4, pp. 1-16, 2020.

[16] S. Belmor, C. Ravichandran, and F. Jarad, "Nonlinear generalized fractional differential equations with generalized fractional integral conditions," Journal of Taibah University for Science, vol. 14, no. 1, pp. 114-123, 2020.

[17] A. P. Chen and Y. Chen, "Existence of solutions to antiperiodic boundary value problem for nonlinear fractional differential equations with impulses," Advances in Difference Equations, vol. 2011, Article ID 915689, 17 pages, 2011.

[18] J. V. Devi and Ch.V. Sreedhar, "Generalized monotone iterative method for caputo fractional integro-differential equations," European Journal of Pure and Applied Mathematics, vol. 9, no. 4, pp. 346-359, 2016.

[19] S. Karthikeyan, C. Ravichandran, and T. Gunasekar, "Existence results for hadamard type fractional functional integrodifferential equations with integral boundary conditions," International Journal of Applied Engineering Research, vol. 10, no. 3, pp. 6919-6932, 2015.

[20] X. P. Li, F. L. Chen, and X. Z. Li, "Generalized anti-periodic boundary value problems of impulsive fractional differential equations," Communications in Nonlinear Science and $\mathrm{Nu}$ merical Simulation, vol. 18, pp. 28-41, 2013.

[21] B. Ahmad and J. J. Nieto, "Existence and approximation of solutions for a class of nonlinear impulsive functional differential equations with anti-periodic boundary conditions," Nonlinear Analysis: Real World Applications, vol. 69, no. 10, pp. 3291-3298, 2008.

[22] Z. Luo and J. J. Nieto, "New results for the periodic boundary value problem for impulsive integro-differential equaitons," Communications in Nonlinear Science and Numerical Simulation, vol. 70, pp. 2248-2260, 2009.
[23] L. Chen and J. Sun, "Nonlinear boundary value problem for first order impulsive integro-differential equations of mixed type," Journal of Mathematical Analysis and Applications, vol. 325, no. 2, pp. 830-842, 2007.

[24] M. Rangasamy, R. Poongodi, and S. Dhanalakshmi, "Existence of global solutions for second order impulsive abstract functional integro differential equations in Banach space," International Journal of Mathematical Archive, vol. 4, pp. 182-195, 2013.

[25] G.-X. Song and X.-L. Zhu, "Extremal solutions of periodic boundary value problems for first order integro-differential equations of mixed type," Journal of Mathematical Analysis and Applications, vol. 300, no. 1, pp. 1-11, 2004.

[26] X. Wang and J. Zhang, "Impulsive anti-periodic boundary value problem of first-order integro-differential equations," Journal of Computational and Applied Mathematics, vol. 234, no. 12, pp. 3261-3267, 2010.

[27] G. S. Ladde, V. Lakshmikantham, and A. S. Vatsala, Monotone Iterative Techniques for Nonlinear Differential Equations, Pitman, London, UK, 1985.

[28] V. Lakshmikantham and A. S. Vatsala, Generalizaed Quasilinesrization for Nonlinear Problems, Kluwer Academic Publishers, Berlin, Germany, 1998.

[29] V. B. Mandelzweig and F. Tabakin, "Quasilinearization approach to nonlinear problems in physics with application to nonlinear ODEs," Computer Physics Communications, vol. 141, no. 2, pp. 268-281, 2001.

[30] B. Ahmad, "A quasilinearization method for a class of integrodifferential equations with mixed nonlinearities," Nonlinear Analysis: Real World Applications, vol. 7, pp. 997-1004, 2006.

[31] P. G. Wang, C. R. Li, J. Zhang, and T. X. Li, "Quasilinearization method for first-order impulsive integro-differential equations," Electronic Journal of Differential Equations, vol. 46, pp. 1-14, 2019.

[32] P. G. Wang, Y. M. Wang, C. M. Jiang, and T. X. Li, "Convergence of solutions for functional integro-differential equations with nonlinear boundary conditions," Advances in Difference Equations, vol. 2019, no. 4, pp. 1-16, 2019.

[33] A. Cabada and J. J. Nieto, "Rapid convergence of the iterative technique for first order initial value problems," Applied Mathematics and Computation, vol. 87, no. 2-3, pp. 217-226, 1997.

[34] T. G. Melton and A. S. Vatsala, "Generalized quasilinearization and higher order of convergence for first order initial value problems," Dynamic Systems and Applications, vol. 15, pp. 375-394, 2006.

[35] T. G. Melton and A. S. Vatsala, "Higher order convergence via generalized quasilinearization method for impulsive differential equations," International Journal of Mathematical Archive, vol. 1186, pp. 284-291, 2009.

[36] R. N. Mohapatra, K. Vajravelu, and Y. Yin, "Extension of the method of quasilinearization and rapid convergence," Journal of Optimization Theory and Applications, vol. 96, no. 3, pp. 667-682, 1998.

[37] H. Wang and W. Yi, "Multilinear singular and fractional integral operators on weighted morrey spaces," Journal of Function Spaces and Applications, vol. 2013, no. 5, Article ID 793263, 11 pages, 2013.

[38] P. G. Wang and X. Liu, "Rapid convergence of approximate solutions for singular differential systems," Electronic Journal of Differential Equations, vol. 203, pp. 1-12, 2015.

[39] Y. Yin, "Monotone iterative technique and quasilinearization for some anti-periodic problem," Nonlinear World, vol. 3, no. 2, pp. 253-266, 1996. 